\title{
Development of an optimal protocol for isolation and purification of human granulosa cells in patients with different ovarian reserves
}

\author{
YING HAN, GE GAO, SHUANG LI, NAN XIAO, YINFENG ZHANG and HAINING LUO \\ Department of Center for Reproductive Medicine, Tianjin Central Hospital of Obstetrics and Gynecology, \\ Tianjin 300100, P.R. China
}

Received October 30, 2020; Accepted May 28, 2021

DOI: $10.3892 / \mathrm{etm} .2021 .10370$

\begin{abstract}
The aim of the current study was to develop an accurate and reproducible method for isolation of granulosa cells (GCs) in patients with different ovarian reserves. The cells of healthy individuals and patients with polycystic ovary syndrome (PCOS) were isolated using a modified two-step Percoll density gradient centrifugation. The cells of patients with poor ovarian response (POR) were isolated using a one-step method suitable for samples containing few cells. Cells extracted using these purification techniques were compared regarding cell yield, viability and purity using immunocytochemistry, flow cytometry, Cell Counting Kit-8, western blotting and RNA integrity analysis. The purity and activity of the cells in the POR group were comparable with those in the healthy and PCOS groups and no statistically significant differences were identified. Furthermore, isolated cells analyzed for RNA integrity indicated good quality RNA and presented an RNA integrity number of 8-10, demonstrating that the technique enabled the isolation of GCs from different types of patients. Thus, a reliable and reproducible technique for the isolation of pure GCs with high yield is described in the present study. This protocol provides an efficient method targeted to patients with different ovarian reserve functions that enables the preparation of GCs for evaluating their molecular functions.
\end{abstract}

\section{Introduction}

Granulosa cell (GC) apoptosis and/or premature removal of surrounding GCs deprive oocytes of growth factors, nutrients and survival factors resulting in apoptosis in diplotene-arrested

Correspondence to: Dr Haining Luo, Department of Center for Reproductive Medicine, Tianjin Central Hospital of Obstetrics and Gynecology, 156 Nankai Sanma Road, Nankai, Tianjin 300100, P.R. China

E-mail: luohn163@163.com

Key words: granulosa cell, poor ovarian response, polycystic ovary syndrome, ovarian reserve function, RNA integrity oocytes cultured in vitro (1), as demonstrated by Tiwari et al (2). Therefore, GC apoptosis in ovulated cumulus-oocyte complexes can be used as predictor of oocyte quality (3). Establishing appropriate in vitro GC models is important in the study of ovarian function and assists in elucidation of the mitogenic, luteotropic and apoptotic mechanisms responsible for selection and support of the dominant ovarian follicle (4). Furthermore, successful cell isolation and purification methods are essential for accurate interpretation of experimental data. A common source of human GCs is from follicular fluid from patients undergoing in vitro fertilization (IVF) procedures (5). Therefore, further high-volume, high-quality studies aimed at separating GCs from follicular fluid that are high quality and sufficient in number is essential (6). Several human GC isolation techniques have been described in the literature (7-12). The density gradient procedure is a rapid, simple and relatively inexpensive technique. In addition, it allows the recovery of a high percentage of luteinized GCs (11). Sun et al (12) confirmed the efficacy of two-step 50\% Percoll gradient centrifugation to separate and extract GCs. However, to the best of our knowledge, there are no existing studies that compare the efficacy of a method of isolating GCs in patients with varying ovarian reserve function. The purification method for human GCs described in the current study utilizes a Percoll gradient to remove contaminating red blood cells, followed by the selection of GC aggregates. This technique was tailored to produce a pure population of GCs from patients with varying ovarian reserve function, in order to obtain high-quality RNA for gene expression studies. The process of the purification and characterization of GCs has been summarized in Fig. 1. Thus, the aim of the current study was to provide an efficient method of preparing GCs from patients with varying ovarian reserve functions in order to evaluate their molecular function.

\section{Materials and methods}

Patients and sample preparation. Between December 2015 and June 2016, infertile women who were eligible for IVF/intracytoplasmic sperm injection (ICSI) treatment at the Reproductive Medicine Centre of Tianjin Central Hospital of Obstetrics and Gynecology were examined for eligibility and those that consented to participate in the present study were recruited. The study was approved by the hospital's 
Institutional Review Board (approval no. TJCOB2016002) and was performed in accordance with the Declaration of Helsinki. No additional interventions were included in the routine clinical and laboratory standards for IVF/ICSI preparation and treatment. Informed written consent was obtained from all participants. The exclusion criteria were as follows: Diabetes type 1 or 2 , impaired thyroid, renal or hepatic function, congenital adrenal hyperplasia, endometriosis and hypothalamic amenorrhea.

Table I summarizes data from a cohort of 45 patients who underwent oocyte retrieval. A consensus was reached on the minimal criteria needed to define poor ovarian response (POR) and polycystic ovary syndrome (PCOS). POR was defined in accordance with the Bologna criteria (13). At least two of the following three features had to be present to be categorized as POR: i) Advanced maternal age ( $\geq 40$ years) or any other risk factor for POR; ii) previous POR ( $\leq 3$ oocytes with a conventional stimulation protocol); iii) an abnormal ovarian reserve test [for example, antral follicle count, 5-7 follicles or anti-Müllerian hormone (AMH), 0.5-1.1 ng/ml].

Only subjects with a diagnosis of PCOS according to the Rotterdam criteria (14) were included and one of the features had to be polycystic ovaries on ultrasound examination. At least two of the following three features had to be present to be categorized as PCOS: i) Oligo- or anovulation; ii) clinical and/or biochemical signs of hyperandrogenism; iii) polycystic ovaries and exclusion of other etiologies (congenital adrenal hyperplasia, androgen-secreting tumors, Cushing's syndrome).

\section{Cell culture and reagents}

Healthy individuals and PCOS patients. Follicular granulosa-luteal cells were obtained after oocyte retrieval from 38 patients undergoing IVF treatment at the Reproductive Medicine Centre of Tianjin Central Hospital of Obstetrics and Gynecology. All patients participating in the present study underwent controlled ovarian stimulation according to routine long or short gonadotropin-releasing hormone agonist protocols. The follicular aspirates from each patient were pooled in conical bottomed 15-ml polypropylene centrifuge tubes. These were then centrifuged at $800 \mathrm{x} g$ for $10 \mathrm{~min}$ at room temperature after which the supernatant was discarded. After adding a small quantity of saline to resuspend the cells, the cell suspension was transferred to an equal volume of $50 \%$ (v/v) Percoll (Sigma-Aldrich; Merck KGaA) by centrifugation at $500 \mathrm{x} g$ for $20 \mathrm{~min}$ at room temperature. After resuspending the cells using saline, they were separated with an equal volume of $0.25 \%$ trypsin digestion solution(Sigma-Aldrich; Merck KGaA) for $10 \mathrm{~min}$ at room temperature. Then, after the addition of the same volume of Gibco DMEM/F-12 media (Thermo Fisher Scientific, Inc.) with $10 \%$ fetal bovine serum (ES-Cult ${ }^{\mathrm{TM}}$ FBS; Stemcell Technologies, Inc.) to neutralize trypsin, a centrifugation was performed at $800 \mathrm{x}$ g for $5 \mathrm{~min}$ at room temperature and density gradient centrifugation was repeated. Then, to perform a second density gradient centrifugation, the supernatant was discarded, and the cells were mixed well with normal saline and then centrifuged at $500 \mathrm{x} \mathrm{g}$ for $20 \mathrm{~min}$ at room temperature in an equal volume of $50 \%$ Percoll. GCs were carefully aspirated with pipettes, mixed well with saline and centrifuged at $800 \mathrm{x} g$ for $5 \mathrm{~min}$ at room temperature, after which the supernatant was discarded.

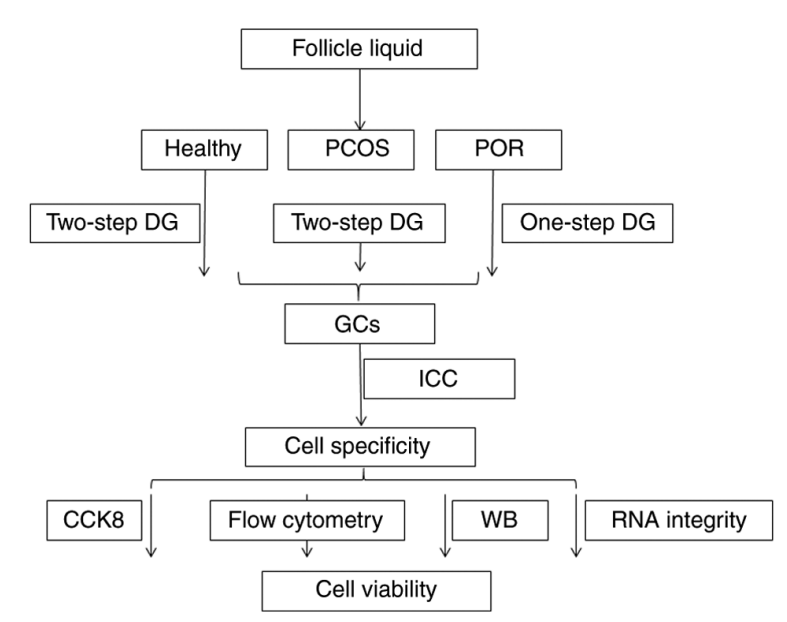

Figure 1. Representative diagram of the steps involved in purification and characterization of GCs. PCOS, polycystic ovary syndrome; POR, poor ovarian response; DG, density gradient; GCs, granulosa cells; ; CCK8, Cell Counting Kit-8; WB, western blot.

The collected human GCs were cultured in DMEM/F12 containing $10 \% \mathrm{FBS}$ and $100 \mathrm{U} / \mathrm{ml}$ penicillin/streptomycin. Samples were taken for cell counting, viability testing via Trypan blue exclusion, and immunocytochemistry and western blot analysis. The remainder was cultured in an incubator under a constant temperature of $37^{\circ} \mathrm{C}$ and $5 \% \mathrm{CO}_{2}$. Nine samples were processed for RNA extraction to analyze the integrity.

POR patients. The follicular aspirates from seven POR patients were pooled. These were then centrifuged at $800 \mathrm{x} g$ for $10 \mathrm{~min}$ at room temperature after which the supernatant was discarded. After resuspending cells using saline, they were separated using an equal volume of $0.25 \%$ trypsin for digestion at room temperature for $10 \mathrm{~min}$. Subsequent processing was consistent with the abovementioned protocol. One sample was processed for RNA extraction to analyze the integrity.

Immunocytochemistry. GCs were seeded at $70 \%$ confluence onto small glass coverslips placed into 24 -well plates. After $24 \mathrm{~h}$ of culture the coverslips were removed, washed with PBS three times, fixed with $100 \%$ methanol $\left(-20^{\circ} \mathrm{C}\right)$ for $15 \mathrm{~min}$ at room temperature, washed with PBST three times, and blocked for $1 \mathrm{~h}$ with $5 \%$ (v/v) goat serum (Gibco; Thermo Fisher Scientific, Inc.) in PBS. Next, the cells were incubated overnight at $4{ }^{\circ} \mathrm{C}$ with the following primary antibodies: Polyclonal goat anti-human $\mathrm{IgG}$ follicle stimulating hormone receptor (FSHR; 1:100; cat. no. sc-7798) and polyclonal rabbit anti-human IgG to Müllerian inhibiting substance type II receptor (MISIIR; 1:200; cat. no. sc-67287) from Santa Cruz Biotechnology, Inc., and then incubated with secondary antibody rabbit anti-goat $\mathrm{IgG}$ and goat anti-rabbit $\mathrm{IgG}$ (1:200; cat. no. sc2768 and sc2004) at room temperature for $1 \mathrm{~h}$ and rinsed with PBS. Chromogen 3,3'-diaminobenzidine tetrachloride (SERVA Electrophoresis $\mathrm{GmbH}$ ) was used as a substrate. The cell nucleus was stained with Harris hematoxylin solution (cat. no. HY-N0116; MedChemExpress) for $15 \mathrm{~min}$ at room temperature. The expression levels of FSHR and MISIIR were scored according to the extent and intensity of 
Table I. Patient characteristics in the three groups.

\begin{tabular}{lccc}
\hline & \multicolumn{3}{c}{ Subject } \\
\cline { 2 - 4 } & $\begin{array}{c}\text { Patients with } \\
\text { POR } \\
\text { Characteristic }\end{array}$ & $\begin{array}{c}\text { Healthy } \\
\text { individuals } \\
(\mathrm{n}=5)\end{array}$ & $\begin{array}{c}\text { Patients with } \\
\text { PCOS } \\
(\mathrm{n}=22)\end{array}$ \\
\hline Age, years & $37 \pm 3.16$ & $29 \pm 3.22$ & $27 \pm 3.26$ \\
No. of oocytes & $5.4 \pm 1.14$ & $16.6 \pm 2.81$ & $25.7 \pm 5.26$ \\
retrieved & & & \\
Basal FSH & $10.7 \pm 2.45$ & $6.1 \pm 1.10$ & $4.5 \pm 1.19$ \\
Basal FSH/LH & $2.7 \pm 1.15$ & $1.4 \pm 0.99$ & $0.6 \pm 0.74$ \\
\hline
\end{tabular}

POR, poor ovarian response; PCOS, polycystic ovary syndrome; FSH, follicle-stimulating hormone; $\mathrm{LH}$, luteinizing hormone.

staining. The extent of staining was scored by the percentage of the positively stained area in each region of interest using the following scale: 0 for $<5 \%$; 1 for $5-25 \%$; 2 for $25-50 \%$; 3 for $50-75 \%$; and 4 for $\geq 75 \%$ (15). The staining intensity was scored as 0, 1, 2 and 3 for the representation of negative (no staining), mild (weak), intermediate (distinct) and intense (strong) staining, respectively (15). The staining intensity and stained area percentage were multiplied to establish a weighted score. Scoring was determined by three independent evaluators without any knowledge of the pathological and clinical characteristics of the patients.

Determination of the percentage of GCs by flow cytometry. To confirm their phenotype, the GCs were characterized for the expression of specific surface antigens defining human GCs. Cells incubated for $30 \mathrm{~min}$ at $4^{\circ} \mathrm{C}$ with 1:200 diluted human FSHR-phycoerythrin (PE) from R\&D Systems, Inc., (cat. no. FAB65591P), CD9-PE (cat. no. 312105; BioLegend, Inc.) and CD24-PE (cat. no. 12-0247-42; eBioscience; Thermo Fisher Scientific, Inc.). After staining, cells were mixed well with PBS and cell fluorescence was evaluated in 10,000 viable cells using a BD FACSCanto ${ }^{\mathrm{TM}}$ II flow cytometer (BD Biosciences).

Cell viability measurement. Cells were seeded in multi-well plates (96-well) in DMEM/F12 medium containing 10\% FBS, at a density of $1 \times 10^{4}$ cells/well, and allowed to attach to the DMEM/F12 medium containing 10\% FBS for $8 \mathrm{~h}$ following analysis. Cells were counted using Cell Counting Kit-8 (CCK-8; Dojindo Molecular Technologies, Inc.) according to the manufacturer's protocol, and the absorbance was measured at $450 \mathrm{~nm}$. The cell viability was analyzed using enzyme-linked immunosorbent assay and optical density values were read at $450 \mathrm{~nm}$. The survival rate was calculated with the following formula: [(As-Ab)/(Ac-Ab)] x100\% (16), where As is the absorbance of PCOS and POR groups; Ab is the absorbance of the blank group, which contains complete medium without cells; and Ac is the healthy group.

Measurement of GC apoptosis by flow cytometry. Cell lines were seeded into 12 -well plates and cultured at $37^{\circ} \mathrm{C}$ for $72 \mathrm{~h}$.
The cells were collected into centrifuge tubes, washed three times with PBS and stained with propidium iodide (PI) and FITC-Annexin V (AnV) from the FITC Annexin V Apoptosis Detection kit I (BD Biosciences), followed by flow cytometry using the BD FACSCanto ${ }^{\mathrm{TM}}$ II. The percentage of the cell population in the process of apoptosis was calculated using FlowJo software (v10.4; FlowJo LLC).

Western blot analysis. The total protein from GCs was collected using RIPA buffer (Sangon Biotech Co., Ltd.). A total of $1 \mathrm{ml}$ RIPA buffer was used per $100 \mathrm{mg}$. Cells were collected from a 6 -well plate using $100 \mu \mathrm{l}$ RIPA buffer per well. The lysates were homogenized and then centrifuged at $16,000 \mathrm{x}$ g for $30 \mathrm{~min}$ at $4^{\circ} \mathrm{C}$. The membrane protein was extracted using the Membrane and Cytoplasm Protein Extraction Kit (Nanjing KeyGen Biotech Co., Ltd.) according to the manufacturer's instructions. Proteins from the supernatant were quantified using a BCA assay (Thermo Fisher Scientific, Inc.), an equal volume of $1 \mathrm{X}$ loading buffer was added and then the protein was denatured for $10 \mathrm{~min}$ at $100^{\circ} \mathrm{C}$. A total of $20 \mu \mathrm{g}$ protein from each sample was separated on a $10 \%$ SDS-PAGE gel and transferred to a PVDF membrane (Bio-Rad Laboratories, Inc.). The membranes were blocked for $1 \mathrm{~h}$ at room temperature in 5\% non-fat milk in Tris-buffered saline with $0.1 \%$ Tween-20. Subsequently, the membranes were incubated overnight at $4^{\circ} \mathrm{C}$ with 1:1,000 diluted goat anti-FSHR (cat. no. sc-7798; Santa Cruz, Biotechnology, Inc.), 1:1,000 diluted rabbit anti-caspase-3 (cat. no. sc-65497; Santa Cruz Biotechnology, Inc.), 1:1,000 diluted rabbit anti-Bcl-2 (cat. no. sc-7382; Santa Cruz Biotechnology, Inc.) and 1:10,000 diluted mouse anti- $\beta$-actin (cat. no. C1213; Sungene Biotech, Ltd.). The next day, the membranes were incubated with the corresponding secondary antibodies rabbit anti-goat $\mathrm{IgG}$, goat anti-rabbit IgG and goat anti-mouse (1:200; cat. no. sc2768, sc2004 and sc2005, respectively) at 1:1,000 for $1 \mathrm{~h}$ at room temperature. The immune complexes were examined by ECL detection (EMD Millipore). The western blotting bands were quantified using ImageJ Software (v1.46; National Institutes of Health) after background subtraction.

RNA extraction and amplification. For RNA analysis, GC samples were obtained from: Patients with $\operatorname{PCOS}(n=5)$; healthy patients $(n=2)$; and patients with POR $(n=2)$. Total RNA was extracted using an RNAequeous ${ }^{\circledR}$-Micro kit (Ambion; Thermo Fisher Scientific, Inc.) according to the manufacturer's instructions. The samples were analyzed for total RNA concentration using TRIzol ${ }^{\circledR}$ reagent (Invitrogen; Thermo Fisher Scientific, Inc.), and total RNA quality and level of degradation using an Agilent 2100 Bioanalyzer (Agilent Technologies Deutschland $\mathrm{GmbH}$ ) according to the manufacturer's instructions. All of the RNA samples showed two distinct peaks representing $18 \mathrm{~S}$ and $28 \mathrm{~S}$ ribosomal RNA, which indicated good quality RNA, and presented an RIN of 8-10.

Statistical analysis. The data were analyzed using SPSS 19.0 software (IBM Corp.). Student's unpaired t-test was performed for mean comparison between two groups. The comparisons among multiple groups were analyzed using ANOVA followed by the Least significant difference post hoc test. Experimental 

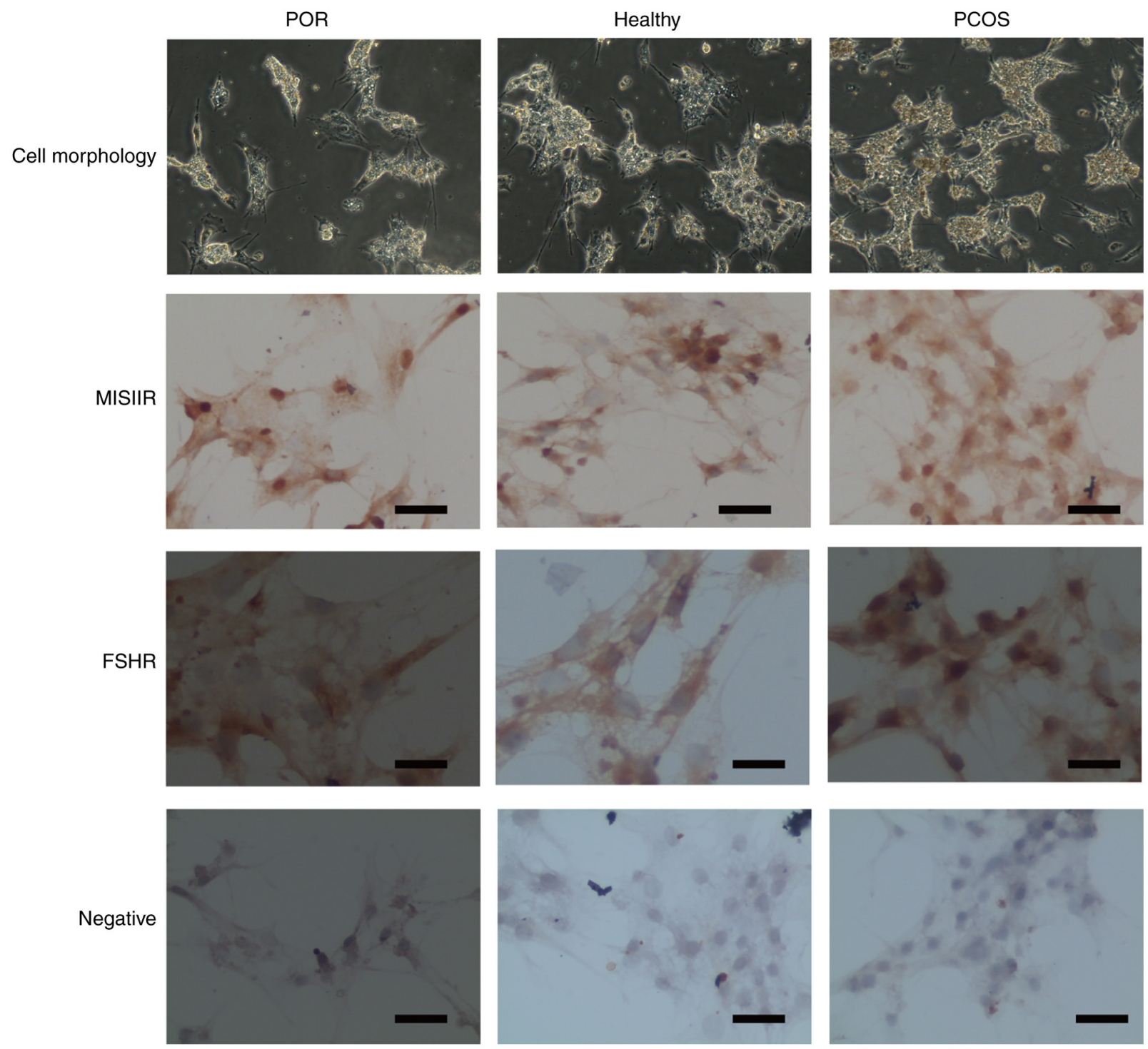

Figure 2. Cell morphology and expression of MISIIR and FSHR in GCs. The top panel demonstrates GCs that were isolated and plated on coverslips in DMEM/F12 for $24 \mathrm{~h}$ (magnification, x20). The subsequent three rows show representative immunocytochemistry staining of MISIIR and FSHR in the GCs of: Patients with POR, healthy individuals and patients with PCOS (magnification, $x 40$ ). The negative control lacked the anti-FSHR and anti-MISIIR antibody and no positive signal was observed. Scale bar, $50 \mu \mathrm{m}$. MISIIR, Müllerian inhibiting substance type II receptor; FSHR, follicle stimulating hormone receptor; GCs, granulosa cells; POR, poor ovarian response; PCOS, polycystic ovary syndrome.

data are presented as means \pm standard deviation and $\mathrm{P}<0.05$ was considered to indicate a statistically significant difference.

\section{Results}

Human GCs express MISIIR and FSHR. Following $24 \mathrm{~h}$ of primary culture, single adherent cells were observed in a distinct area of the culture dish. These cells were rhombic or irregular in shape, inconsistent in size and shape, and had clear boundaries. Additionally, connections between the cells were observed under a microscope (Fig. 2). As all cells plated on the cover slips exhibited MISIIR and FSHR (Fig. 2), immunocytochemistry demonstrated that the majority, if not all, of human GCs express these receptors. The mean purity of GC samples obtained using this method was $>95 \%$.

Human GCs express surface antigens. In the three groups $>95 \%$ of GCs were positive for FSHR (Fig. 3). The CD9 and
CD24 molecules are cell surface glycoproteins, which are also expressed on the GCs surrounding the oocytes. Flow cytometry demonstrated that CD9 is present on the cell surface of, on arithmetic average, $70 \%$ of GCs among the three groups and CD24 is present on the cell surface of $90 \%$ of GCs among the three groups. The expression of CD9 on GCs in patients with PCOS was significantly lower than that in the other groups (Fig. 3).

Proliferation rate of PCOS and healthy GCs are increased compared with the POR group. Cell viability was assessed by CCK- 8 assay. An increase of $\sim 50-70 \%$ in cell viability was observed in the cells over time, with no significant differences identified among the three groups. The histogram in Fig. 4 further illustrates the experimental results. Flow cytometry was then performed to evaluate GC apoptosis and the arithmetic average percentages of cells in two quadrants are presented. The early apoptotic cells $\left(\mathrm{PI}^{-} / \mathrm{AnV}^{+}\right)$and late apoptotic cells 

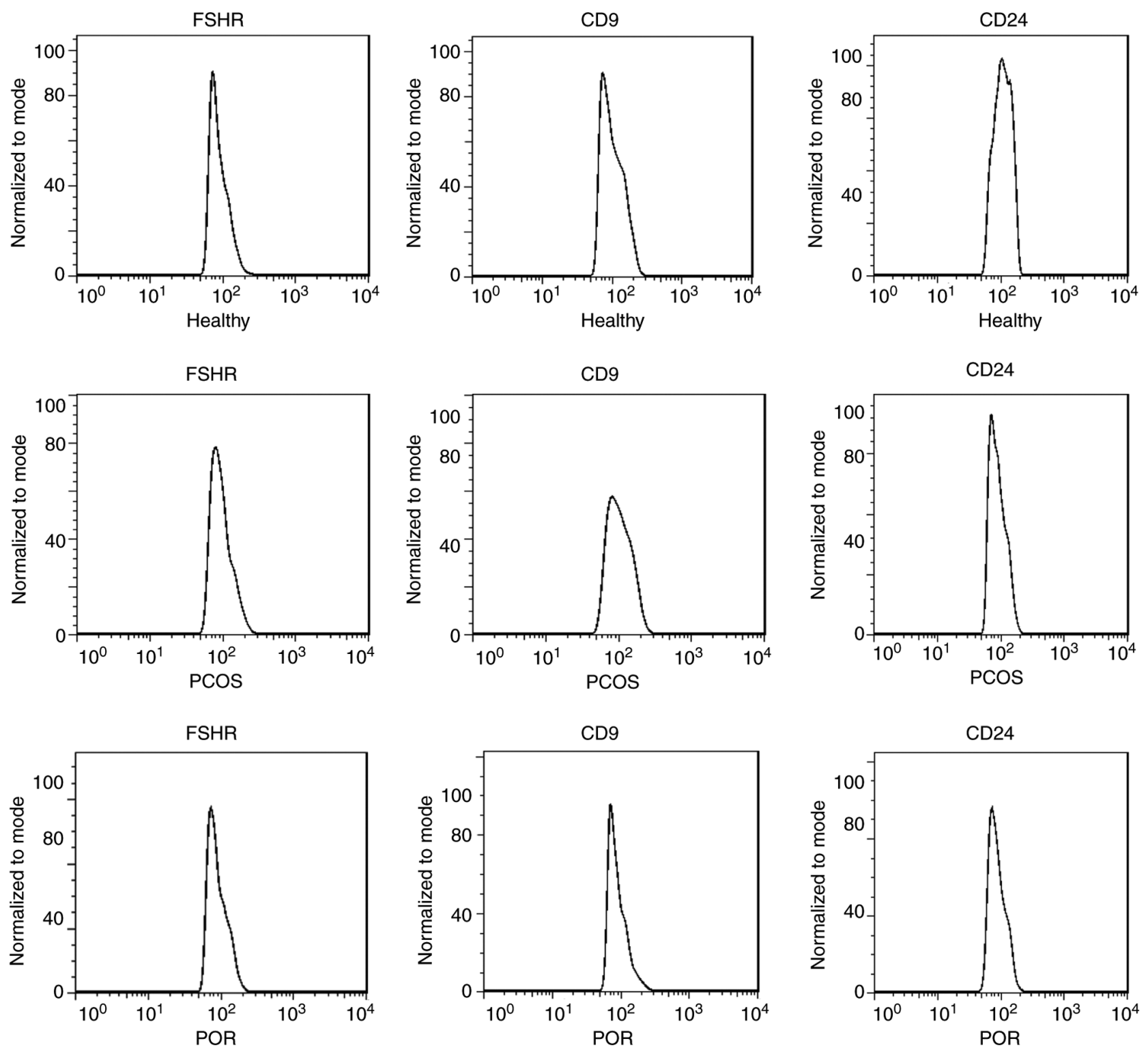

Figure 3. Surface antigen expression of GCs using flow cytometry. In the POR, healthy and PCOS groups, $>95 \%$ of cells were positive for FSHR. CD9 and CD24 were identified on the cell surface of, on arithmetic average, 70 and 90\%, respectively of GCs in the three groups. The expression of CD9 on GCs in patients with PCOS was significantly lower than that in the other groups. GCs, granulosa cells; FSHR, follicle stimulating hormone receptor; POR, poor ovarian response; PCOS, polycystic ovary syndrome.

$\left(\mathrm{PI}^{+} / \mathrm{AnV}^{+}\right)$are presented as percentages of events and no statistically significant differences in the levels of apoptosis were identified among the different groups (Fig. 5). Higher levels of FSHR were observed in the GCs of healthy individuals and patients with PCOS when compared with those of patients with POR ( $\mathrm{P}<0.01$; Fig. 6). The results showed that GCs from healthy individuals and patients with PCOS exhibited increased Bcl-2 expression levels and inhibited early cleavage of caspase-3 when compared with patients with POR.

Analysis of RNA integrity. An RIN was then determined for each sample using an established algorithm following sample assessment in an automated electrophoresis system (Agilent 2100 Bioanalyzer) (17). No statistically significant RNA degradation was observed in the nine samples of the GCs, as demonstrated by the representative electropherograms and corresponding RIN values (Fig. 7). The mean RIN for all patient samples was 9.1. Moreover, the baseline of the electrophoretic pattern was stable, therefore RNA integrity was good, the results were reliable and follow-up studies are now required.

\section{Discussion}

The present study describes a method for purifying human GCs from patients with different ovarian function derived from follicular aspirates. The present study was performed to enable a range of future molecular studies for the analysis of gene expression in order to provide high-quality RNA. As such, a means of purifying GCs was required that maintained RNA integrity and was suitable for all cell types. These aims have been successfully achieved by using the Percoll gradient method described in the current study. In the majority of studies, a Percoll method is used for GC extraction, which separates single GCs and bundles of GCs (6,18-20). Aghadavod et al (6) 

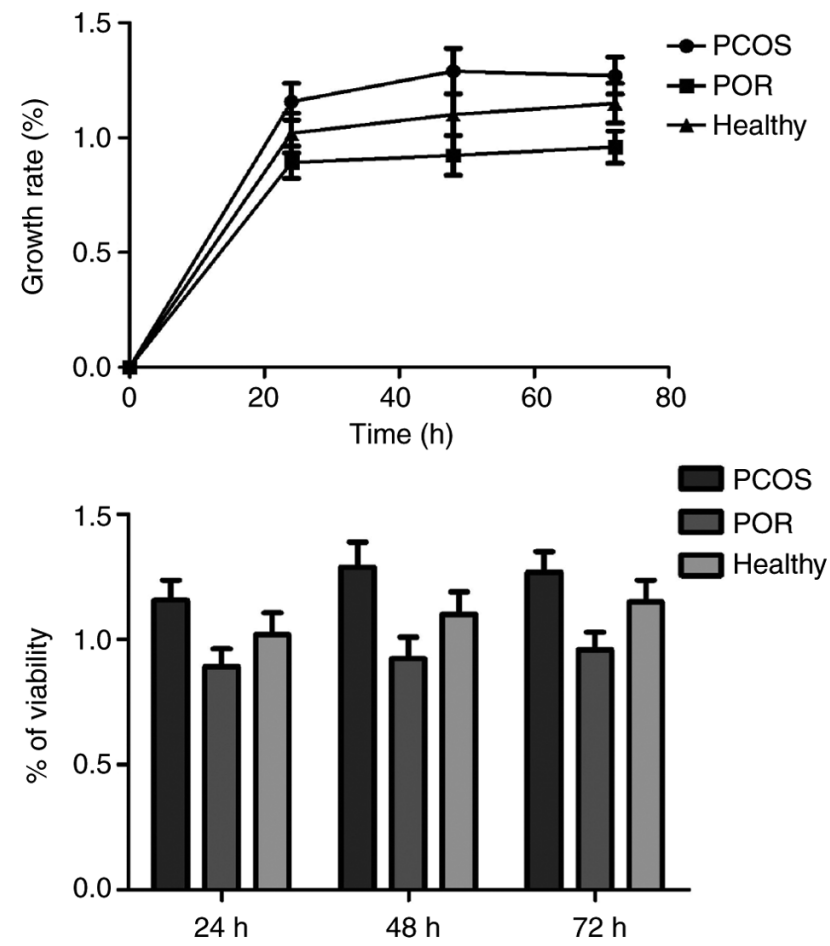

Figure 4. Growth rate of PCOS and POR GCs compared with the healthy group. Cells were cultured in DMEM/F12 medium containing 10\% FBS for $72 \mathrm{~h}$. Then, GC growth rate was evaluated using a Cell Counting Kit- 8 assay. The results are presented as the means \pm SD of three experiments; the data are expressed as percentages. An increase of $\sim 50-70 \%$ in cell viability was observed in cells over time, with no significant differences identified among the three groups. PCOS, polycystic ovary syndrome; POR, poor ovarian response; GCs, granulose cells.

compared Ficoll, Percoll and Red Blood Cell Lysing buffer (RBL) methods for the extraction of GCs and demonstrated that the Percoll method is less likely to cause cellular damage and is more suitable for GC extraction. However, the authors did not evaluate different types of patients.

The developmental capacity of an oocyte is largely dependent on its interaction with GCs through transzonal projections, gap junctions and the secretion of paracrine factors (21). Therefore, the study of GCs is a good starting point when examining oocyte maturation. The specificity of cells cultured in vitro should first be identified; some data indicate that GCs specifically express FSHR as soon as they reach the pre-antral follicle stage (22) and secrete AMH as soon as they reach the primary follicle stage $(23,24)$. Previous studies identified that CD9 is associated with the integrin $\alpha 6 \beta 1$ involved in GC adhesion and regulation of GC luteinization (25-27). CD9 expression on GCs increases through ovulation, but at ovulation CD9 expression is reduced on GCs of mature follicles when compared with immature follicles (25-27). CD24 is an important mediator of ovulation and CD24(+) GCs are essential for triggering ovulation. Treatment with human chorionic gonadotropin significantly increases the expression of CD24 in GCs $(28,29)$. Furthermore, CD9 and CD24 expression on GCs has been linked with female reproductive function (25-29). Clavero et al (26) identified that CD9 may be involved in the regulation of follicular luteinization, CD9 and $\mathrm{CD} 24$ producing an inhibition of the luteinization of GC and preventing premature follicular luteinization. Therefore, in
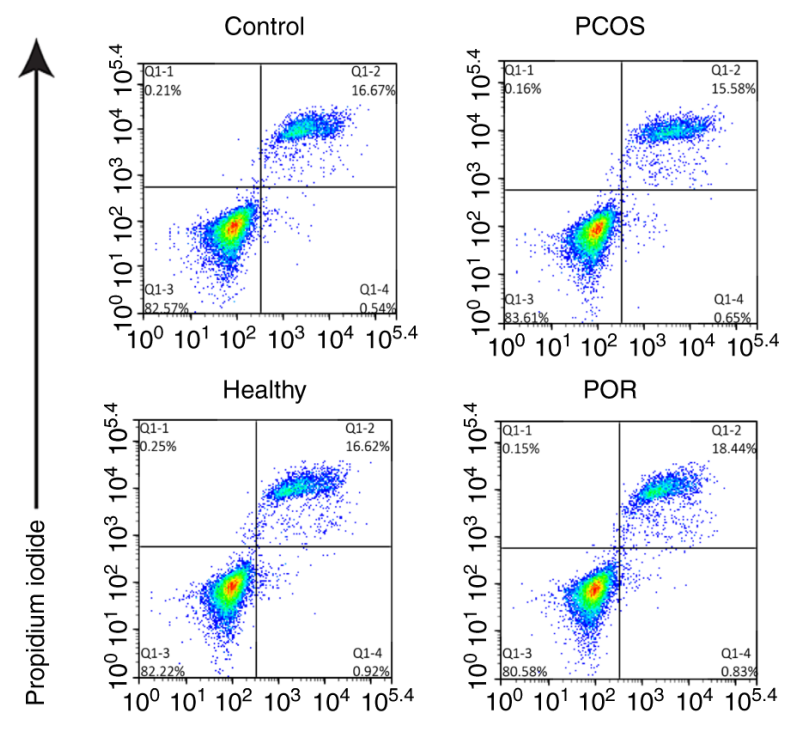

Annexin V-FITC

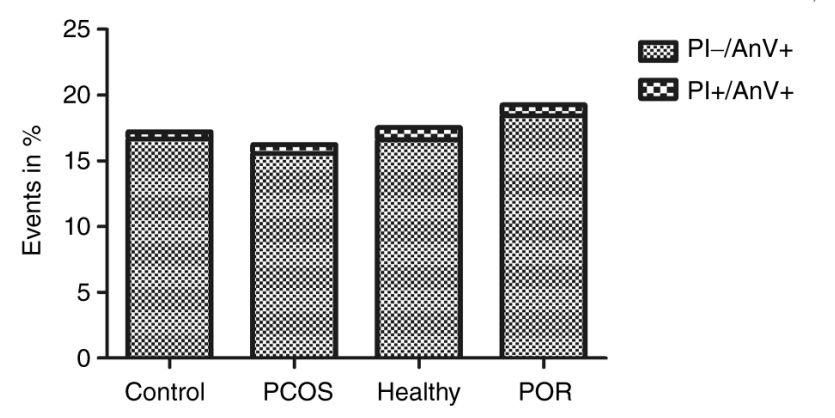

Figure 5. The cells were cultured for $72 \mathrm{~h}$. Both the supernatant and attached cells were harvested and stained with PI and AnV, followed by subsequent FACS analysis. The arithmetic average percentage of cells in two quadrants is presented. Early apoptotic cells $\left(\mathrm{PI}^{-} / \mathrm{AnV}^{+}\right)$and late apoptotic cells $\left(\mathrm{PI}^{+} / \mathrm{AnV}^{+}\right)$ are presented as percentages of events. No statistically significant difference was observed in the levels of apoptosis. PCOS, polycystic ovary syndrome; POR, poor ovarian response; PI, propidium iodide; AnV, Annexin V.

the present study, the expression levels of FSHR and MISIIR served as the standards for the identification of GCs. The results of immunocytochemistry demonstrated that almost all cells plated on the cover slips showed presence of MISIIR and FSHR. Flow cytometry showed that the positive rate of FSHR in cultured GCs reached $>95 \%$ and that CD9 is present on the cell surface of, on arithmetic average, 70\% of GCs among the three groups and CD24 is present on the cell surface of $90 \%$ of GCs among the three groups, which reinforces the credibility of the obtained experimental data. These results suggested that the expression levels of FSHR in humans with different ovarian reserve function gradually decrease with increasing age and degeneration. Thus, the downregulation of CD9 antigen expression in PCOS ovaries would be consistent with arrested folliculogenesis in patients with PCOS (30). The advantage of primary cell culture is to maximize the preservation of cell characteristics, so that in vitro results are more consistent with the environment in vivo.

However, research by Aghadavod et al (6) demonstrated that when GCs were extracted using the RBL method, there were large clumps of aggregated GCs and the number of dead leukocyte and erythrocyte carcasses was negligible. The use of RBL solution can result in more thoroughly dissolved follicular 

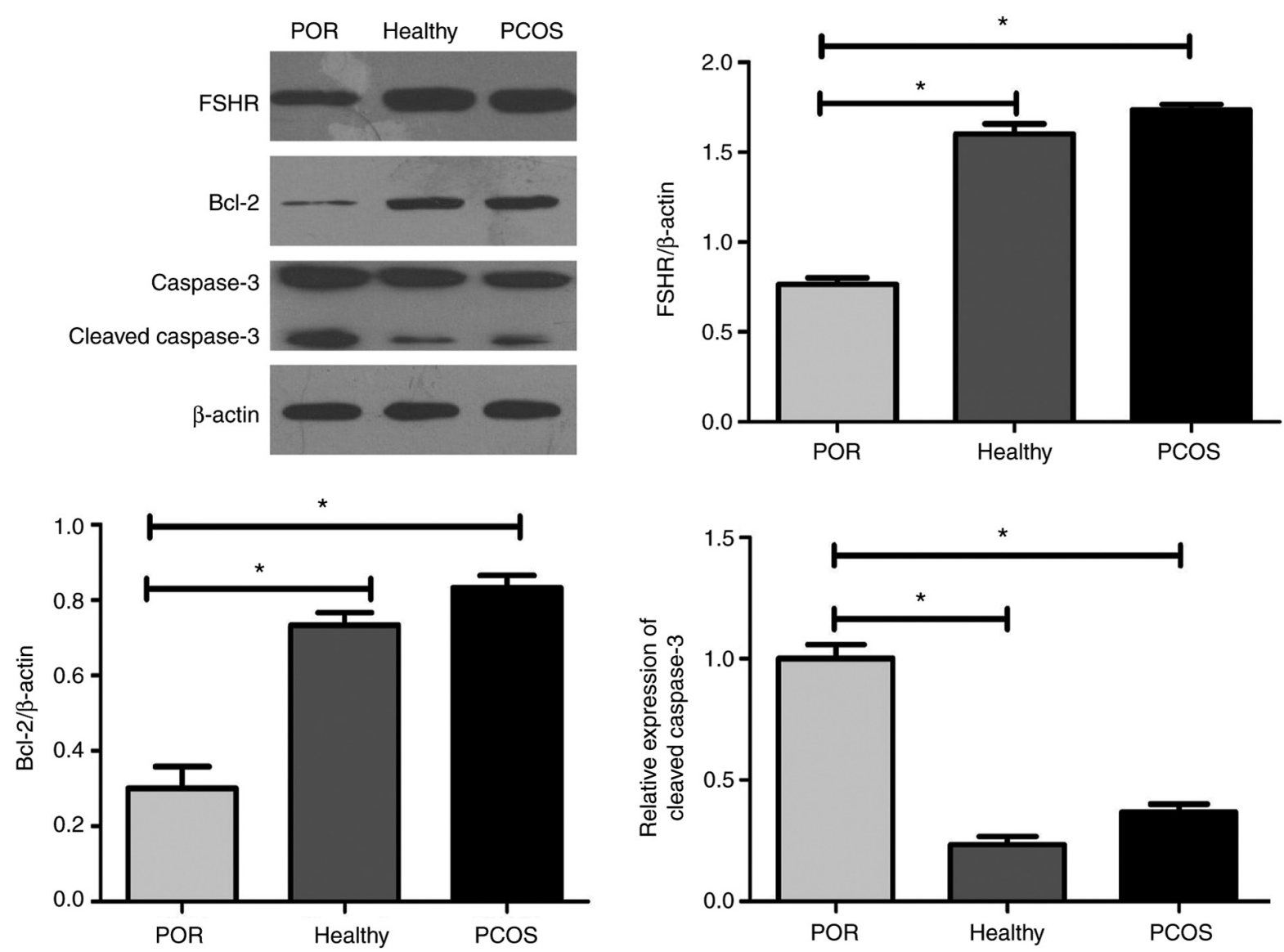

Figure 6. Western blot analysis of the expression of FSHR, Bcl-2 and cleaved caspase-3 proteins in GCs collected from: Patients with POR, healthy individuals and patients with PCOS. The expression was normalized to $\beta$-actin separately. The bar charts illustrate the outcomes of western blot analyses. Data are representative of three independent experiments and were calculated as the means \pm SD of three experiments. ${ }^{*}<<0.01$ vs. POR patients. FSHR, follicle stimulating hormone receptor; GCs, granulosa cells; POR, poor ovarian response; PCOS, polycystic ovary syndrome.

fluid mixed with red blood cells, but a larger quantity of cell debris was apparent in the GCs in our preliminary experiment. In the method used in the current study, the small quantity of red blood cell residues did not significantly affect the adherence rate of GCs cultured at $24 \mathrm{~h}$. In the present study, the red blood cells were removed using the direct Percoll density gradient separation method and two-step centrifugation and, thus, ensured cell purity. Follicular fluid often contains white mucus, which supports and protects oocytes after the removal of cumulus-GC clusters (5). Following centrifugation, mucus is located in the upper layer of erythrocyte sedimentation and under microscopic examination, a large number of cumulus GCs are observed (5). In the study by Sun et al (12), the gradient centrifugation method combined with trypsin digestion was used to separate human GCs, which also removes other mixed cells and tissue fragments and helps to obtain higher purity ovarian GCs. Liu et al (31) confirmed that the cell clusters were significantly reduced after trypsin digestion and the cells were easier to adhere to. The cells then grew well and were evenly distributed (31). The present study used trypsin to digest the extracellular matrix components within the mucus. It can be effectively removed from adhering to GCs and therefore significantly improves GC purity.

Due to different ovarian reserve functions, a single method may not be applicable to all types of patients. To the best of our knowledge, there are no studies that compare the efficacy of a method of isolating GCs in patients with different ovarian reserve function. In the current study, cells from healthy individuals and patients with PCOS were isolated using two-step Percoll density gradient centrifugation. Cells from patients with POR were isolated using a one-step method owing to the lower number of cells. The limitation of the current study was that the number of GCs in the follicular fluid of patients with POR was very small due to the two-step centrifugation method. Therefore, the results of GC extraction in patients with POR using the two- and one-step Percoll protocols were not compared. The aim of the present study was to evaluate the efficacy of one-step and two-step DG for the extraction of GCs in patients with different ovarian reserve functions.

Immunocytochemistry and the CCK-8\flow cytometry results demonstrated that the purity and activity of the cells in the POR group were similar to those in the healthy and PCOS groups, and that the observed differences were not statistically significant. RNA integrity in the POR group patients, although slightly lower than the other two groups of patients, was high enough that one step DG could be conducted in follow-up experiments. This extraction method for GCs shows that it is effective in patients with differing ovarian reserve functions.

Decreased ovarian reserve, such as during POR, may be due to ovarian cell apoptosis (32). One of the primary mechanisms of ovarian apoptosis involves mitochondrial apoptotic signaling cascades, which are mediated by mitochondrial 
POR-1

RIN:8.9

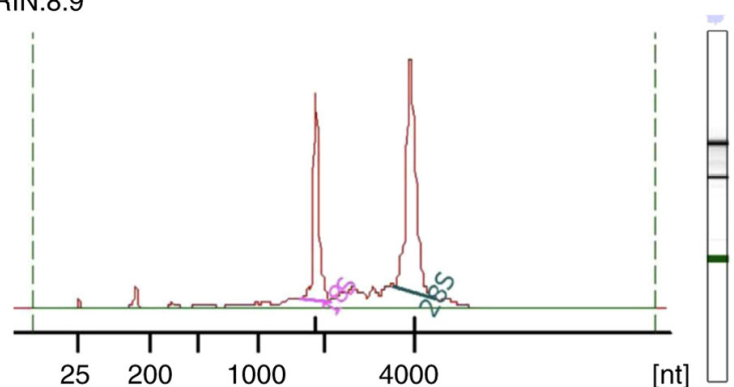

Healthy-1

RIN:9.4

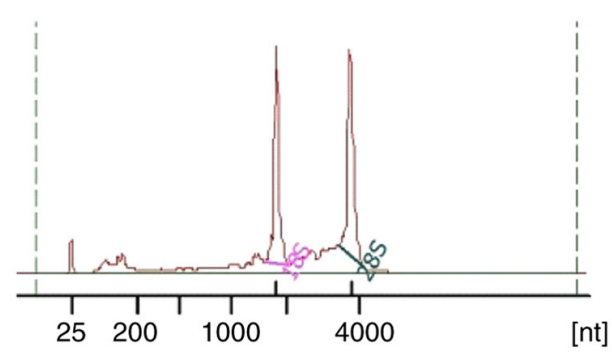

RIN:8.9

PCOS-1

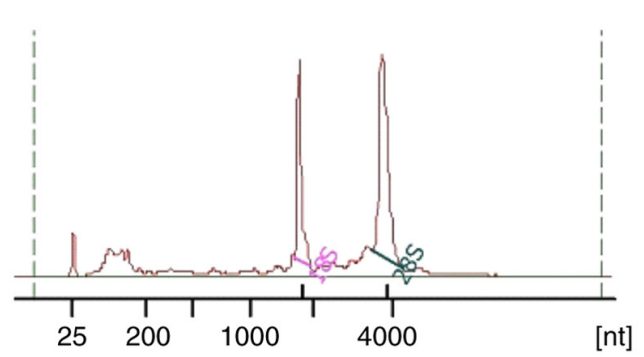

RIN:9.7

PCOS-3
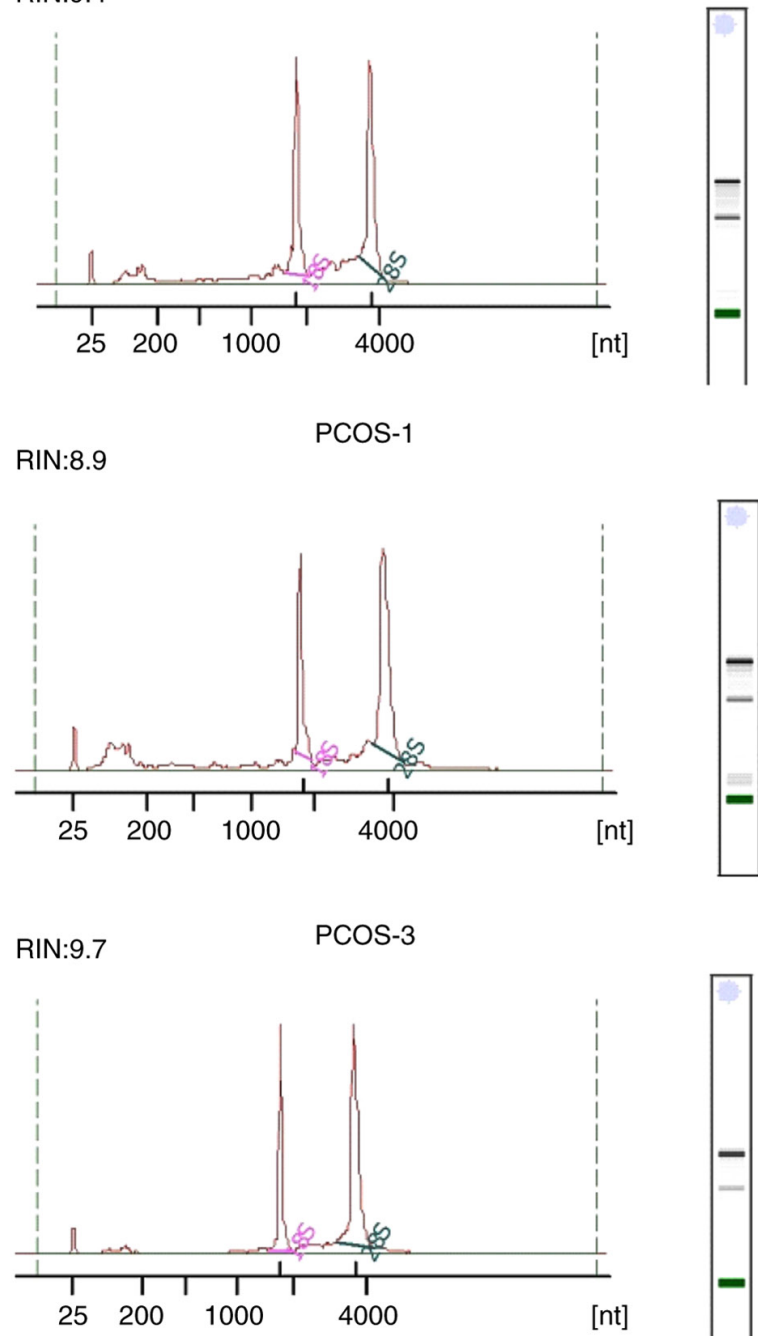

RIN:8.4

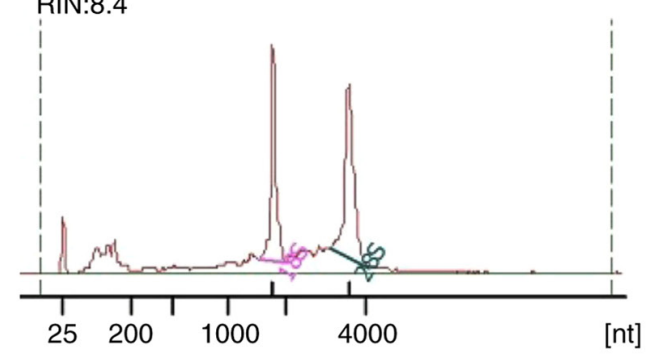

Healthy-2

RIN:9.2

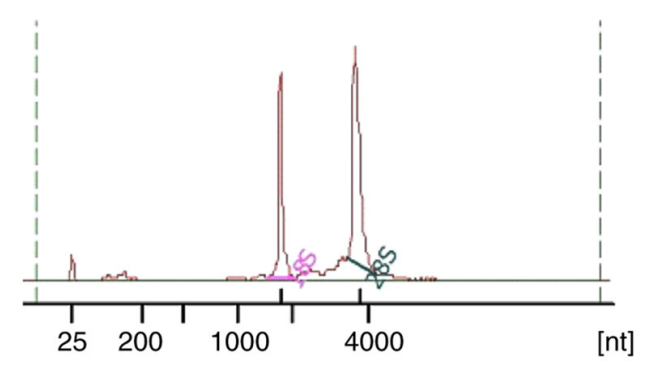

RIN:9.1

PCOS-2
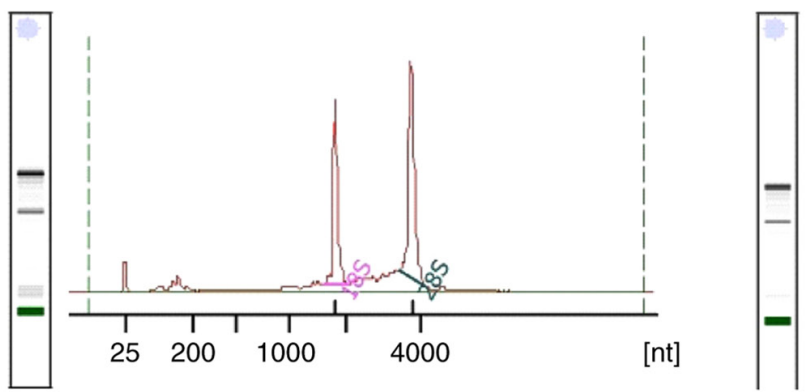

RIN:9.5

PCOS-4
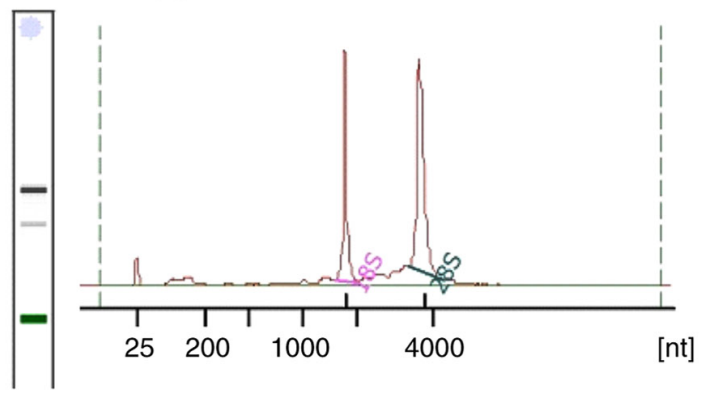
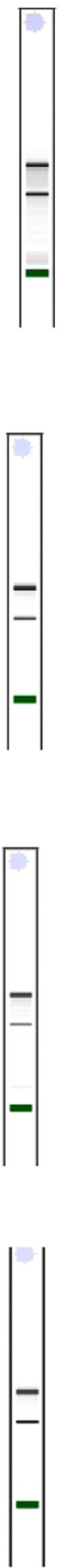

RIN:9.3

PCOS-5
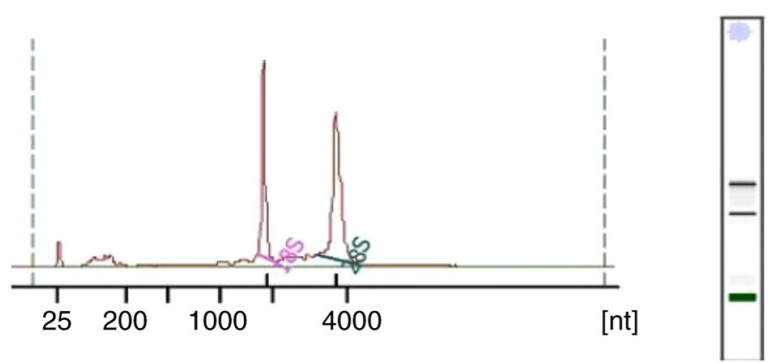

Figure 7. RNA integrity analysis of RNA isolated from GCs was performed using an Agilent 2100 Bioanalyzer. Representative electropherograms for GC samples from: Five patients with PCOS, two healthy individuals and two patients with POR. The RIN is presented for each sample. GCs, granulosa cells; RIN, RNA Integrity Number; PCOS, polycystic ovary syndrome; POR, poor ovarian response. 
Bcl-2 family members (33). In the current study, the expression of Bcl-2 and caspase-3 were compared in three different patient groups. Active caspase- 3 and Bcl-2 expression levels were significantly downregulated in the GCs of patients with PCOS when compared with those of individuals in the POR and healthy groups. This result is consistent with a previous study by Chuffa et al (34).

Thus, the present study demonstrated that the newly modified procedure used in the current study was highly efficient for $\mathrm{GC}$ isolation in patients with differing ovarian reserve functions. Use of this optimized protocol will enable the study of GC complex functions and molecular functions, and extends our knowledge regarding the mechanisms involved in oocyte apoptosis.

\section{Acknowledgements}

Not applicable.

\section{Funding}

The present study was performed with financial support from the Tianjin Health and Family Planning Commission (grant no. 2018007) and the National Natural Science Foundation of China (grant no. 81803927).

\section{Availability of data and materials}

The datasets used and/or analyzed during the current study are available from the corresponding author on reasonable request.

\section{Authors' contributions}

YH conceived and designed the research, and interpreted the results of the experiments. GG contributed to the design of the study and the interpretation of experimental results. GG, SL, NX, YZ and HL performed experiments, analyzed data, prepared figures and drafted the manuscript. GG and $\mathrm{YZ}$ also edited and revised manuscript. YH and HL confirm the authenticity of all the raw data. All authors have read and approved the final version of the manuscript.

\section{Ethics approval and consent to participate}

All procedures performed in studies involving human participants were in accordance with the ethical standards of the Institutional Review Board of Tianjin Central Hospital of Obstetrics and Gynecology (approval no. TJCOB2016002) and were performed in accordance with the Declaration of Helsinki. Informed consent was obtained from all individual participants included in the study.

\section{Patient consent for publication}

Not applicable.

\section{Competing interests}

The authors declare that they have no competing interests.

\section{References}

1. Tiwari M, Prasad S, Tripathi A, Pandey AN, Ali I, Singh AK, Shrivastav TG and Chaube SK: Apoptosis in mammalian oocytes: A review. Apoptosis 20: 1019-1025, 2015.

2. Tiwari M, Tripathi A and Chaube SK: Presence of encircling granulosa cells protects against oxidative stress-induced apoptosis in rat eggs cultured in vitro. Apoptosis 22: 98-107, 2017.

3. Levay PF, Huyser C, Fourie FL and Rossouw DJ: The detection of blood contamination in human follicular fluid. J Assist Reprod Genet 14: 212-217, 1997.

4. Havelock JC, Rainey WE and Carr BR: Ovarian granulosa cell lines. Mol Cell Endocrinol 228: 67-78, 2004.

5. Chilvers RA, Bodenburg YH, Denner LA and Urban RJ: Development of a novel protocol for isolation and purification of human granulosa cells. J Assist Reprod Genet 29: 547-556, 2012.

6. Aghadavod E, Zarghami N, Farzadi L, Zare M, Barzegari A, Movassaghpour AA and Nouri M: Isolation of granulosa cells from follicular fluid; applications in biomedical and molecular biology experiments. Adv Biomed Res 4: 250, 2015.

7. Asem EK, Feng S, Stingley-Salazar SR, Turek JJ, Peter AT and Robinson JP: Basal lamina of avian ovarian follicle: Influence on morphology of granulosa cells in-vitro. Comp Biochem Physiol C Toxicol Pharmacol 125: 189-201, 2000.

8. Lobb DK and Younglai EV: A simplified method for preparing IVF granulosa cells for culture. J Assist Reprod Genet 23: 93-95, 2006.

9. Grondahl ML, Borup R, Lee YB, Myrhoj V, Meinertz H and Sorensen S: Differences in gene expression of granulosa cells from women undergoing controlled ovarian hyperstimulation with either recombinant follicle-stimulating hormone or highly purified human menopausal gonadotropin. Fertil Steril 91: 1820-1830, 2009.

10. Ferrero H, Delgado-Rosas F, Garcia-Pascual CM, Monterde M, Zimmermann RC, Simón C, Pellicer A and Gómez R: Efficiency and purity provided by the existing methods for the isolation of luteinized granulosa cells: A comparative study. Hum Reprod 27: 1781-1789, 2012

11. Raad G, Bazzi M, Tanios J, Mourad Y, Azouri J, Azouri J and Fakih C: Optimization of the cell aggregates method for isolation and purification of human granulosa cells from follicular fluid. Int J Fertil Steril 13: 339-345, 2020.

12. Sun Y,Lin Y,Li H,Liu J, Sheng X and Zhang W: 2,5-Hexanedione induces human ovarian granulosa cell apoptosis through BCL-2, BAX, and CASPASE-3 signaling pathways. Arch Toxicol 86: 205-215, 2012.

13. Ferraretti AP, La Marca A, Fauser BC, Tarlatzis B, Nargund G and Gianaroli L: ESHRE consensus on the definition of "poor response' to ovarian stimulation for in vitro fertilization: The Bologna criteria. Hum Reprod 26: 1616-1624, 2011.

14. Rotterdam ESHRE/ASRM-Sponsored PCOS Consensus Workshop Group: Revised 2003 consensus on diagnostic criteria and long-term health risks related to polycystic ovary syndrome. Fertil Steril 81: 19-25, 2004.

15. Wei W, Sun HH, Li N, Li HY, Li X, Li Q and Shen XH: WNT5A modulates cell cycle progression and contributes to the chemoresistance in pancreatic cancer cells. Hepatobiliary Pancreat Dis Int 13: 529-538, 2014.

16. Giovannetti E, Mey V, Danesi R, Mosca I and Del Tacca M: Synergistic cytotoxicity and pharmacogenetics of gemcitabine and pemetrexed combination in pancreatic cancer cell lines. Clin Cancer Res 10: 2936-2943, 2004.

17. Schroeder A, Mueller O, Stocker S, Salowsky R, Leiber M, Gassmann M, Lightfoot S, Menzel W, Granzow M and Ragg T: The RIN: An RNA integrity number for assigning integrity values to RNA measurements. BMC Mol Biol 7: 3, 2006.

18. Chin EC, Harris TE and Abayasekara DR: Changes in cAMP-dependent protein kinase (PKA) and progesterone secretion in luteinizing human granulosa cells. J Endocrinol 183: 39-50, 2004.

19. Shahnazi V, Zaree M, Nouri M, Mehrzad-Sadaghiani M, Fayezi S, Darabi M, Khani S and Darabi M: Influence of $\omega-3$ fatty acid eicosapentaenoic acid on IGF-1 and COX-2 gene expression in granulosa cells of PCOS women. Iran J Reprod Med 13: 71-78, 2015.

20. Motta PM, Nottola SA, Familiari G, Makabe S, Stallone T and Macchiarelli G: Morphodynamics of the follicular-luteal complex during early ovarian development and reproductive life. Int Rev Cytol 223: 177-288, 2003. 
21. Gilchrist RB, Lane M and Thompson JG: Oocyte-secreted factors: Regulators of cumulus cell function and oocyte quality. Hum Reprod Update 14: 159-177, 2008.

22. Gougeon A: Regulation of ovarian follicular development in primates: Facts and hypotheses. Endocr Rev 17: 121-155, 1996.

23. Durlinger AL, Visser JA and Themmen AP: Regulation of ovarian function: The role of anti-Müllerian hormone. Reproduction 124: 601-609, 2002.

24. Weenen C, Laven JS, Von Bergh AR, Cranfield M, Groome NP, Visser JA, Kramer P, Fauser BC and Themmen AP: Anti-Müllerian hormone expression pattern in the human ovary: Potential implications for initial and cyclic follicle recruitment. Mol Hum Reprod 10: 77-83, 2004.

25. Takao Y, Fujiwara H, Yamada S, Hirano T, Maeda M, Fujii S and Ueda M: CD9 is expressed on the cell surface of human granulosa cells and associated with integrin alpha6beta1. Mol Hum Reprod 5: 303-310, 1999.

26. Clavero A, Castilla JA, Martinez L, Mendoza N, Fontes J and Maldonado V: Expression of integrin fraction and adhesion molecules on human granulosa cells and its relation with oocyte maturity and follicular steroidogenesis. J Assist Reprod Genet 21: 187-195, 2004

27. Jaslow CR, Patterson KS, Cholera S, Jennings LK, Ke RW and Kutteh WH: CD9 Expression by human granulosa cells and platelets as a predictor of fertilization success during IVF. Obstet Gynecol Int 2010: 192461, 2010.
28. Wissing ML, Kristensen SG, Andersen CY, Mikkelsen AL, Høst T, Borup R and Grøndahl ML: Identification of new ovulation-related genes in humans by comparing the transcriptome of granulosa cells before and after ovulation triggering in the same controlled ovarian stimulation cycle. Hum Reprod 29: 997-1010, 2014.

29. Dong JP, Dai ZH, Jiang ZX, He Y, Wang L, Liao QY, Sun NX, Wang YN, Sun SH, Lin W, et al: CD24: A marker of granulosa cell subpopulation and a mediator of ovulation. Cell Death Dis 10: 791, 2019.

30. Oksjoki S, Söderström M, Inki P, Vuorio E and Anttila L: Molecular profiling of polycystic ovaries for markers of cell invasion and matrix turnover. Fertil Steril 83: 937-944, 2005.

31. Liu Y, Chen X, Xue X, Shen C, Shi C, Dong J, Zhang H, Liang R, $\mathrm{Li} \mathrm{S}$ and $\mathrm{Xu}$ J: Effects of Smad3 on the proliferation and steroidogenesis in human ovarian luteinized granulosa cells. IUBMB Life 66: 424-437, 2014.

32. Seifer DB, Gardiner AC, Ferreira KA and Peluso JJ: Apoptosis as a function of ovarian reserve in women undergoing in vitro fertilization. Fertil Steril 66: 593-598, 1996.

33. Hussein MR, Haemel AK and Wood GS: Apoptosis and melanoma: Molecular mechanisms. J Pathol 199: 275-288, 2003.

34. Chuffa LG, Lupi Júnior LA and da Maia Lima AF: Sex steroid receptors and apoptosis-related proteins are differentially expressed in polycystic ovaries of adult dogs. Tissue Cell 48: 10-17, 2016. 